Virginia Commonwealth University

VCU Scholars Compass

2010

\title{
Magnetism in $\mathrm{ZnO}$ nanowire with Fe/Co codoping: First-principles density functional calculations
}

\section{S. Ghosh}

Indian Association for the Cultivation of Science

Q.Wang

Virginia Commonwealth University

G. P. Das

Indian Association for the Cultivation of Science

Puru Jena

Virginia Commonwealth University, pjena@vcu.edu

Follow this and additional works at: http://scholarscompass.vcu.edu/phys_pubs

Part of the Physics Commons

Ghosh, S., Wang, Q. Das, G.P., et al. Magnetism in ZnO nanowire with Fe/Co codoping: First-principles density functional calculations. Physical Review B, 81, 235215 (2010). Copyright (C) 2010 American Physical Society.

\section{Downloaded from}

http://scholarscompass.vcu.edu/phys_pubs/65

This Article is brought to you for free and open access by the Dept. of Physics at VCU Scholars Compass. It has been accepted for inclusion in Physics Publications by an authorized administrator of VCU Scholars Compass. For more information, please contact libcompass@vcu.edu. 


\title{
Magnetism in $\mathrm{ZnO}$ nanowire with Fe/Co codoping: First-principles density functional calculations
}

\author{
S. Ghosh, ${ }^{1, *}$ Q. Wang, ${ }^{2}$ G. P. Das, ${ }^{1}$ and P. Jena ${ }^{2}$ \\ ${ }^{1}$ Department of Materials Science, Indian Association for the Cultivation of Science, Jadavpur, Kolkata 700032, India \\ ${ }^{2}$ Department of Physics, Virginia Commonwealth University, Richmond, Virginia 23284, USA
}

(Received 14 April 2010; published 23 June 2010)

\begin{abstract}
Using first-principles density functional calculations, at both generalized gradient approximation (GGA) and $\mathrm{GGA}+U$ levels we have investigated the electronic structure and magnetic properties of Fe/Co codoped $\mathrm{ZnO}$ nanowire. Here we have addressed some of the key issues such as, the preferable sites that Fe/Co can occupy, the coupling mechanism, and role of defects in coupling. We found that the spin alignment between the transition-metal atoms depends on their location. When Fe and Co atoms are nearest neighbors on the outer surface of the nanowire along [0001] direction is the lowest energy configuration with ferrimagnetic (FiM) ground state. At GGA level of description ferromagnetic ordering is observed when impurity atoms sit at surface and subsurface interface forming Fe-O-Co magnetic path, however at GGA $+U$ level of description antiferromagnetic superexchange interaction dominates and all configuration leads to FiM ground state. $\mathrm{GGA}+U$ are found to give more realistic description of electronics structure of Fe/Co codoped $\mathrm{ZnO}$ nanowire. Interestingly $\mathrm{Fe}-\mathrm{V}_{\mathrm{O}}$-Co defect configurations formed by removing the $\mathrm{O}$ atom from $\mathrm{Fe}-\mathrm{O}-\mathrm{Co}$ magnetic path are ferromagnetic when Fe-Co separation is less than 2.596 at GGA and $2.801 \AA$ at GGA $+U$ irrespective of the location of transition ions. We have also found that $\mathrm{Co}$ atom has a tendency to form clusters around $\mathrm{Fe}$ atom leading to inhomogeneous doping concentrations. O vacancy is found to be crucial in case of promoting ferromagnetism in this system. Two competing factors are the Ruderman-Kittel-Kasuya-Yosida (RKKY) type of exchange interaction in bulk environment due to $\mathrm{O}$ vacancy and direct exchange interaction of carriers due to $\mathrm{Fe}-\mathrm{V}_{\mathrm{O}}-\mathrm{Co}$ defect configuration on the surface.
\end{abstract}

DOI: 10.1103/PhysRevB.81.235215 PACS number(s): 75.75. - c, 36.40.Cg, 73.22.-f, 75.50.Pp

\section{INTRODUCTION}

Spintronics is currently an active area of research because spin-based multifunctional electronic device has several advantages over the conventional charge-based devices regarding data-processing speed, nonvolatility, higher integration densities, etc. ${ }^{1}$ The impending need to obtain such device has led to growing interest in developing and designing spintronic materials. Dilute magnetic semiconductors are promising candidates in this regard. In particular, $\mathrm{ZnO}$-based materials have been receiving considerable attention due to its abundance and environment-friendly nature and also due to its potential as a suitable optoelectronic material ${ }^{2,3}$ with a wide band gap $(\sim 3.34 \mathrm{eV})$ and high excitation binding energy of $60 \mathrm{meV}$. The magnetic, electronic, optical, and electrochemical properties of $\mathrm{ZnO}$ offer the unique possibility to create multifunctional integrated device for sensing, processing, and actuating functions in one monolithic structure. With the advancement of experimental techniques interest in zero-dimensional and one-dimensional (1D) ZnO-based materials as building blocks of new electronic and spintronic devices is rapidly increasing. The study of the effect of dimensionality on magnetic, optical, and mechanical properties is therefore important not only for technological applications but also to strengthen our basic understanding in low dimensions.

Recently transition-metal (TM) doped 1D ZnO nanowires of varying diameter were grown by various experimental techniques and ferromagnetic (FM) ordering has been found at a range of temperatures from low to room temperature. ${ }^{4-9}$ Considerable effort has also been devoted for introducing two different $3 d$-TM impurities into $\mathrm{ZnO}$ matrix in order to explore the effect of codoping on transition temperature. Cho et $a l .{ }^{10}$ reported room-temperature ferromagnetism in $\mathrm{ZnO}$ films codoped with $\mathrm{Fe}$ and $\mathrm{Co}(\mathrm{Fe} / \mathrm{Co})$, which were prepared using a reactive magnetron cosputtering technique. Liu et $a l .{ }^{6}$ successfully synthesized $(\mathrm{Fe} / \mathrm{Co})$ codoped $1 \mathrm{D} \mathrm{ZnO}$ naowires via chemical vapor deposition growth method and observed Curie temperature was higher than $300 \mathrm{~K}$. Previous theoretical study by Park and $\mathrm{Min}^{11}$ on bulk $\mathrm{Zn}_{1-\mathrm{x}}(\mathrm{FeCo})_{\mathrm{x}} \mathrm{O}$ indicated that there is no indication of charge transfer between $\mathrm{Fe}$ and Co. They suggested that double exchange mechanism will not be effective for observed ferromagnetism and one needs to invoke other exchange mechanism between Fe and Co. Defects such as O vacancy or $\mathrm{Zn}$ interstitial ( $n$-type defect) and Zn vacancy ( $p$-type defect) seem to play important role and need to be investigated.

We have carried out first-principles investigation of electronic structure and magnetic properties of $(\mathrm{Fe} / \mathrm{Co})$ codoped $\mathrm{ZnO}$ nanowire. Here we have addressed some of the key issues such as (a) what are the preferable sites that $\mathrm{Fe}$ and $\mathrm{Co}$ atoms occupy? (b) What is the appropriate coupling mechanism between $\mathrm{Fe}$ and $\mathrm{Co}$ ? (c) What is the role of defects in the coupling between $\mathrm{Fe}$ and $\mathrm{Co}$ ? The present results also demonstrate the role of surface curvature and the radial confinement of the electrons on magnetic coupling in codoped $\mathrm{ZnO}$ nanowires.

\section{THEORETICAL METHOD}

The $\mathrm{ZnO}$ nanowire has been constructed from a $(7 \times 7$ $\times 2) \mathrm{ZnO}$ wurtzite supercell by removing $\mathrm{Zn}$ and $\mathrm{O}$ atoms from outside the circle of $1 \mathrm{~nm}$ diameter and replacing it with vacuum space shown in Fig. 1(a). The nanowire thus 
(a)

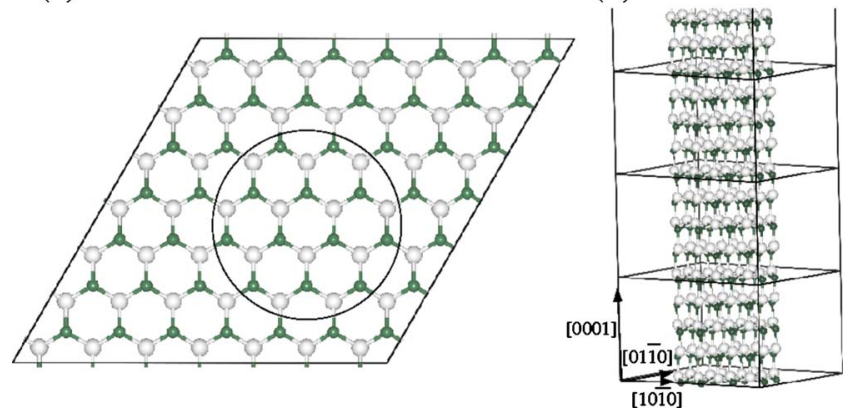

FIG. 1. (Color online) (a) Top view of a $7 \times 7 \times 2 \mathrm{ZnO}$ supercell (0001) plane having wurtzite structure. (b) A $\mathrm{ZnO}$ nanowire supercell $\left(\mathrm{Zn}_{48} \mathrm{O}_{48}\right)$ which extends to infinite along the [0001] direction. The white (lighter) spheres are $\mathrm{Zn}$ and dark green (gray) small spheres are $\mathrm{O}$.

created contains 96 atoms $\left(\mathrm{Zn}_{48} \mathrm{O}_{48}\right)$ with a vacuum space of 12.997 and $13.011 \AA$ along the $[10 \overline{1} 0]$ and [01 $\overline{1} 0]$ directions, respectively, thereby ensuring that wires in neighboring supercells do not interact with reach other. Along the [0001] direction the wire extends to infinity through Bloch periodicity as shown in Fig. 1(b). ${ }^{12}$

In order to simulate codoping, we have replaced two $\mathrm{Zn}$ atoms with one $\mathrm{Fe}$ and one $\mathrm{Co}$ atom which corresponds to $\sim 2 \% \mathrm{Fe}$ and $\sim 2 \%$ Co, i.e., $\sim 4 \% \mathrm{Fe} / \mathrm{Co}$ codoped $\mathrm{ZnO}$ nanowire. Since the preferred sites for $\mathrm{Fe}$ and $\mathrm{Co}$ atoms are not known a priori, we have tried 14 different configurations that can be categorized into four groups: for Group-I, where both $\mathrm{Fe}$ and $\mathrm{Co}$ are in the bulk environment, i.e., having tetrahedral coordination. Group-II, where both $\mathrm{Fe}$ and Co lie on the cylindrical surface, i.e., having one broken bond. Group-III, where $\mathrm{Fe}$ lies on the surface and $\mathrm{Co}$ is at the subsurface and Group-IV, where Co lies on the surface and $\mathrm{Fe}$ at the subsurface. Figure 2 shows these four categories, Group-I and Group-II, each having three possible configurations and

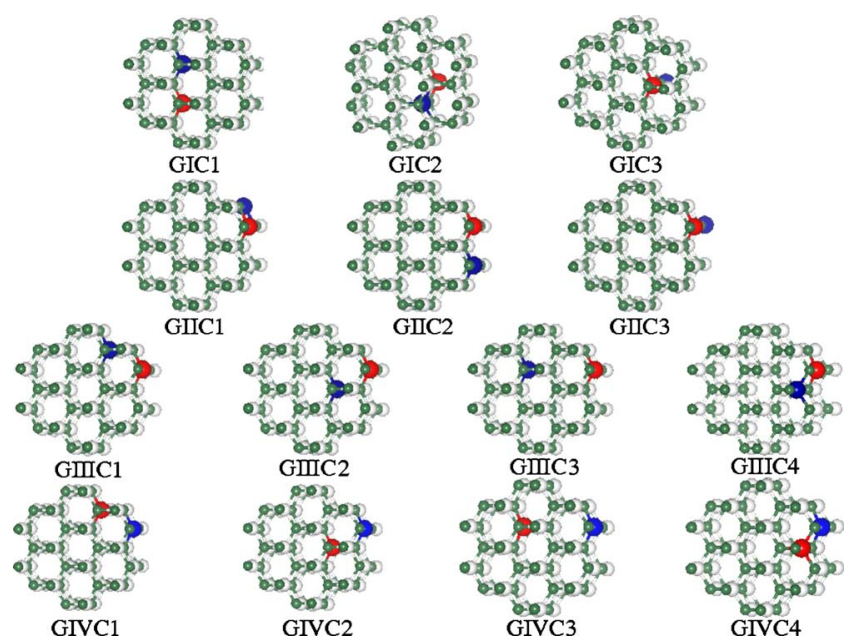

FIG. 2. (Color online) The schematic representation of 14 configurations of $\mathrm{Zn}_{46} \mathrm{Fe}_{1} \mathrm{Co}_{1} \mathrm{O}_{48}$ nanowire supercell. The white (lighter) spheres are $\mathrm{Zn}$, dark green (gray) small spheres are $\mathrm{O}$, the red (gray) large spheres are $\mathrm{Fe}$, and the blue (dark gray) large spheres are Co.
Group-III and Group-IV having four possible configurations leading to a total of 14 configurations. These configurations simulate different possible $\mathrm{Fe}-\mathrm{Co}$ and $\mathrm{Fe}-\mathrm{O}-\mathrm{Co}$ distances as well as Fe-O-Co bond angles. In Group-III and Group-IV we have taken into account the situations where $\mathrm{Fe}$ and $\mathrm{Co}$ occupy the surface and the subsurface (in GIII) and vice versa (in GIV). Here we have not considered the effect of surface passivation, i.e., the surface have dangling bonds when we have determined the preferable sites and magnetic coupling between TM atoms.

For each of these configurations we have computed the total energies corresponding to both FM and ferrimagnetic (FiM) alignments of $\mathrm{Fe}$ and Co spins with full geometry optimization without any symmetry constraint. We have used spin polarized density functional theory ${ }^{13}$ and generalized gradient approximation (GGA) (Ref. 14) for exchange and correlation. The calculations were carried out using the $\mathrm{Vi}$ enna $a b$ initio simulation package (VASP) and plane-wave basis set. ${ }^{15}$ The projector augmented wave (PAW) potentials ${ }^{16}$ were used for $\mathrm{Zn}, \mathrm{O}, \mathrm{Fe}$, and Co. These potentials are known to be more accurate than the conventional or ultrasoft pseudopotentials in treating magnetic systems involving transition metals. The energy cutoff is set to $350 \mathrm{eV}$. Here we have taken soft oxygen PAW potential (PAW_GGA O_s 04May1998) where recommended minimum cutoff for oxygen is $250 \mathrm{eV}$. The accuracy of our calculations for $\mathrm{ZnO}$ system has been well established from our previous work. $^{12,17,18}$ The convergence in energy and force was set as $10^{-4} \mathrm{eV} / \AA$ and $10^{-3} \mathrm{eV} / \AA$, respectively.

We approximated the exchange-correlation function with local spin density approximation (LSDA) and LSDA $+U$. It is well documented that LSDA underestimates the band gap, in case of $\mathrm{ZnO}$ despite having an experimental band gap of $3.34 \mathrm{eV}$, LSDA band gap are around $0.8 \mathrm{eV}$. This underestimation of band gap and absence $d$ - $d$ static correlation can lead to an uncorrected metallic description in case of TM doped semiconducting oxides. Recently some attempts were made to revisit $\mathrm{TM}$ doped $\mathrm{ZnO}$ with band-gap correction. Lany et al. ${ }^{19}$ have achieved self-consistence band-gap correction by adding to the standard GGA $+U$ Hamiltonian empirical nonlocal external potentials that depended on atomic type and the angular momentum. They pointed out that GGA and there $+U$ extensions predict the absence of FM coupling in case of $\mathrm{Co}$ doped $\mathrm{ZnO}$ with electron doping where such coupling is expected or may predict FM coupling in case of $\mathrm{Cr}$ doped $\mathrm{ZnO}$ where such coupling may not be possible. When the band gap is corrected it turns out that both Co and $\mathrm{Cr}$ doped $\mathrm{ZnO}$ electron mediated $\mathrm{FM}$ is possible. In another study by Walsh et al. ${ }^{20}$ investigated Co doped $\mathrm{ZnO}$ with using DFT $+U s / d$ method to correct the band-gap error by applying a Coulomb $U$ on both the $s$ and $d$ orbitals to further raise the band gap to $2.8 \mathrm{eV}$. They concluded that when band gap is corrected which leads to the correct location of unoccupied Co $t_{2 \mathrm{~g}}$ states to the $n$-type region of the conduction of $\mathrm{ZnO}$ and then FM can be achieved by reasonable electron doping concentration.

However in case of nanowire the band gap is expected to be large compare to bulk due to quantum confinement. Our calculated band gap for $\mathrm{ZnO}$ naowire is $1.14 \mathrm{eV}$. Density of states for pure $\mathrm{ZnO}$ nanowire shows the broad $(\sim 4-5 \mathrm{eV})$ 


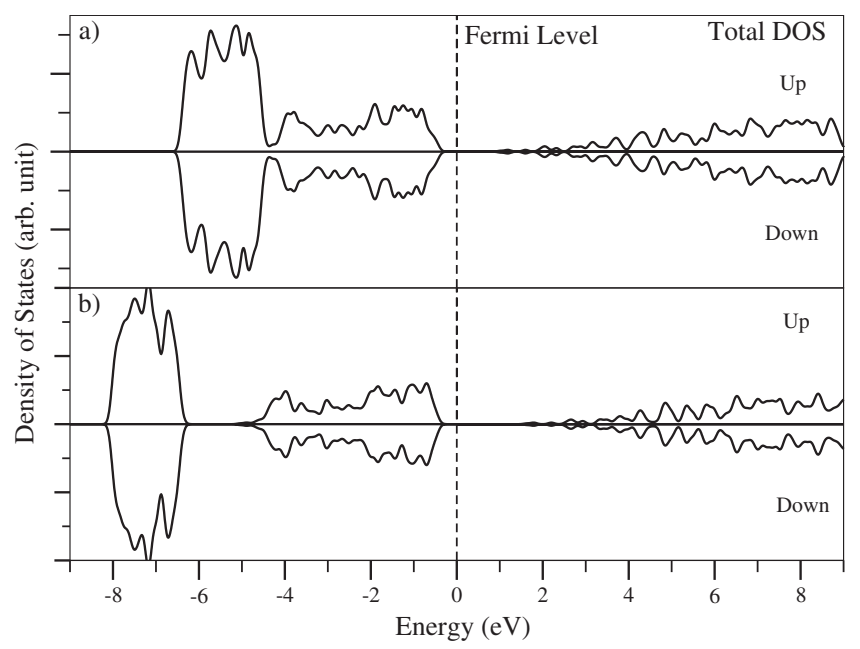

FIG. 3. Total density of states of pure $\mathrm{ZnO}$ nanowire obtained in (a) GGA description and (b) GGA $+U$ description. Applying $U$ $=7 \mathrm{eV}$ and $J=1 \mathrm{eV}$ to $\mathrm{Zn} d$ orbital it becomes more localized $(\sim 7-8 \mathrm{eV})$ and separated from $\mathrm{O} 2 p$ states that has an indirect effect in raising the band gap as it has increased to $2.02 \mathrm{eV}$.

band just below the Fermi level (EF) which is due to $\mathrm{O} 2 p$ states. $\mathrm{Zn} d$ state is just below $\mathrm{O} 2 p$ states and in GGA level overlapping with the $\mathrm{O}$ bands. Applying $U$ $=7 \mathrm{eV}$ and $J=1 \mathrm{eV}$ to $\mathrm{Zn} d$ orbital it becomes more localized $(\sim 7-8 \mathrm{eV})$ and separated from $\mathrm{O} 2 p$ states that has an indirect effect in raising the band gap as it has increased to $2.02 \mathrm{eV}$ (shown in Fig. 3). However it is still below the experimental bulk $\mathrm{ZnO}$ band gap, if we take the band-gap correction in account then the gap in $\mathrm{ZnO}$ nanowire is expected to be larger.

In this paper the computation has been done at both GGA and $\mathrm{GGA}+U_{\mathrm{d}}$ levels. In GGA $+U_{\mathrm{d}}$ calculations we have used rotationally invariant " $+U$ " formulation. We first varied $U$ on both $\mathrm{Co}$ and $\mathrm{Fe}$ from 2 to $6 \mathrm{eV}$ for the ground-state configuration, treating $U$ as parameter, in order to find out how magnetic coupling is affected by the strength of the Coulomb correction. Finally we use $U=3.5 \mathrm{eV}$ for $\mathrm{Fe} d$ and $U$ $=2.8 \mathrm{eV}$ for Co $d$ for all the configurations as these values are reported to give the correct relative stability of different oxide semiconductors ${ }^{19}$ and $U=7 \mathrm{eV}$ is used for $\mathrm{Zn} d$, exchange parameter is set to the typical value of $J=1 \mathrm{eV}$.

\section{RESULTS AND DISCUSSIONS}

We begin with a study of the relaxation of the atoms in the nanowire without $\mathrm{Fe}$ and $\mathrm{Co}$ incorporation. The effects of $\mathrm{Co}$ and $\mathrm{Fe}$ doping on the magnetic properties are then calculated by first keeping the doped nanowire in the unperturbed bulk geometry and then by allowing all the atoms (including $\mathrm{Fe}$ and $\mathrm{Co}$ ) to relax. We find that in pure $\mathrm{ZnO}$ nanowire in wurtzite structure, relaxation of atomic positions is significant due to the large surface area. The total energy of the relaxed cell was found to be $6.712 \mathrm{eV}$ lower in energy than the unrelaxed one at GGA level. This corresponds to an energy gain of $0.14 \mathrm{eV}$ per $\mathrm{Zn}-\mathrm{O}$ dimer. In bulk crystal, the calculated $\mathrm{Zn}-\mathrm{O}$ bond length along the [001] direction is
$1.993 \AA$ while that between $\mathrm{Zn}$ and three $\mathrm{O}$ atoms in (0001) plane is $1.975 \AA$. In $\mathrm{ZnO}$ nanowire, the bond length changed to $1.888 \AA$ for the surface atoms in [0001] direction and to $1.956 \AA$ between the surface and subsurface layer atoms in (0001) plane of the $\mathrm{Zn}_{48} \mathrm{O}_{48}$ supercell. The relaxations of the atoms in the inner sites are much smaller than those on the outermost surface layer, as expected. The total electronic density of states (DOS) for pure $\mathrm{ZnO}$ nanowire using GGA and GGA $+U$, where $U=7 \mathrm{eV}$ is applied on $\mathrm{Zn} d$ orbital are plotted in Fig. 3, which reflects the system is semiconductor and nonmagnetic.

Now we discuss our results for different configurations of the $\mathrm{Fe} / \mathrm{Co}$ codoped $\mathrm{ZnO}$ nanowire, carried out for both FM and FiM states at GGA level. The results are summarized in Table I. The configuration GIIC1, in which Fe and Co atoms are at nearest neighbors on the outer surface layer along [0001] direction of the $\mathrm{ZnO}$ nanowire, is found to be the lowest energy configuration. The difference in FiM and FM total energy $\left(\Delta E=E_{\mathrm{FiM}}-E_{\mathrm{FM}}\right)$ is $-0.175 \mathrm{eV}$, which indicates that the system is in FiM ground state.

Using the ground-state energy of GIIC1 as the reference, we have calculated the relative formation energy $(\Delta \varepsilon)$ of other configurations and found that, GIIC2 and GIVC1 are the next two higher energy configurations with relative formation energies $0.112 \mathrm{eV}$ and $0.122 \mathrm{eV}$, respectively. Here GIIC1 and GIIC2 represent the configuration where Fe and Co both are at surface while GIVC2 represents the configuration where $\mathrm{Co}$ at surface and $\mathrm{Fe}$ at subsurface. The $\mathrm{Fe}-\mathrm{Co}$ distance corresponding to GIIC1, GIIC2, and GIVC1 configurations are $2.60 \AA$, $3.388 \AA$, and $2.478 \AA$, respectively. The corresponding $\Delta E$ values are $-0.175,-0.132$, and 0.119 $\mathrm{eV}$. We also found that when $\mathrm{Fe}$ and $\mathrm{Co}$ atoms are in the bulk environment (GI configurations) and are at surface (GII cofigurations) the magnetic ground state is FiM and at large distance degenerate in energy (DE) while in case when $\mathrm{Fe}$ and $\mathrm{Co}$ atoms are at surface-inner surface environment (GIII and GIV) configurations FM is dominates over FiM. Thus the coupling is dependent not only on the relative separation between Fe-Co but also on the direction and location of the impurity atom, which is a unique manifestation in the nanowire.

In case of unpassivated pure $\mathrm{ZnO}$ naowire the cylindrical surface has dangling bonds. The bonding environment is different when $\mathrm{Zn}$ is replaced on the surface sites and at the subsurface/core site, in this case cylindrical surface has dangling bonds and surface is allowed to relax and then the corresponding energy gain is $0.14 \mathrm{eV}$ per $\mathrm{Zn}-\mathrm{O}$ dimer while in case of passivated pure $\mathrm{ZnO}$ nanowire due to fourfold coordination surface energy minimization will be less and location of the TM atoms in core expected to be enhanced. In case of unpassivated nanowire when $\mathrm{Fe}-\mathrm{Co}$ is on the surface, is allowed to relax to minimize the strain effect and hence the surface substitution is preferred. Looking at the relative formation energies of GIIIC1, GIIIC2 and GIVC1, GIVC2 (given in Table I) it is clear that in case of surfacesubsurface/core interface Co prefers to sit at the surface. The Co-Fe distance is reduced from 2.608 to $2.460 \AA$, when Co is on the surface and $\mathrm{Fe}$ is at the inner site.

In the last three columns of Table I, we have listed the magnetic moments on $\mathrm{Fe}$ and $\mathrm{Co}$ sites along with the in- 
TABLE I. The energy difference $\Delta E$ between FiM and FM states $\left(\Delta E=E_{\mathrm{FiM}}-E_{\mathrm{FM}}\right.$ in eV), the relative energy $\Delta \varepsilon$ (in eV) calculated with respect to the ground-state configuration GIIC1 with geometry optimization, the optimized $\mathrm{Co}-\mathrm{Fe}$ and $\mathrm{Fe}-\mathrm{O}$ and $\mathrm{Co}-\mathrm{O}$ distances (in $\AA$ ) and the magnetic moments (in $\mu_{\mathrm{B}}$ ) at $\mathrm{Fe}$ and $\mathrm{Co}$, and the nearest-neighbor $\mathrm{O}$ atom for the configurations at GGA level given in Fig. 2. Here DE represents degenerate in energy. For DE situation both Fe-O and Co-O distances and magnetic moments on Fe,Co and the nearest-neighbor $\mathrm{O}$ atom are given for both FM and FiM magnetic states.

\begin{tabular}{|c|c|c|c|c|c|c|c|c|c|}
\hline Configurations & $\Delta E$ & $\Delta \varepsilon$ & Coupling & $d_{\mathrm{Fe}-\mathrm{Co}}$ & $d_{\mathrm{Fe}-\mathrm{O}}$ & $d_{\mathrm{Co}-\mathrm{O}}$ & $\mu_{\mathrm{Fe}}$ & $\mu_{\mathrm{Co}}$ & $\mu_{\mathrm{O}}$ \\
\hline GIC1 & -0.065 & 0.671 & FiM & 3.182 & 1.926 & 1.991 & 3.686 & -2.414 & 0.074 \\
\hline GIC2 & -0.014 & 0.688 & FiM & 3.167 & 1.928 & 2.001 & 3.697 & -2.424 & 0.076 \\
\hline \multirow[t]{2}{*}{ GIC3 } & 0.002 & 0.752 & $\mathrm{DE}$ & 5.237 & 1.949 & 1.982 & 3.714 & 2.484 & 0.145 \\
\hline & & & & 5.251 & 1.946 & 1.986 & 3.695 & -2.415 & 0.012 \\
\hline GIIC1 & -0.175 & 0.000 & FiM & 2.605 & 1.847 & 1.803 & 3.220 & -2.269 & -0.066 \\
\hline GIIC2 & -0.132 & 0.112 & FiM & 3.388 & 1.918 & 1.948 & 3.436 & -2.417 & 0.040 \\
\hline \multirow[t]{2}{*}{ GIIC3 } & 0.002 & 0.210 & $\mathrm{DE}$ & 5.140 & 1.869 & 1.828 & 3.500 & 2.456 & 0.165 \\
\hline & & & & 5.130 & 1.868 & 1.827 & 3.50 & -2.452 & 0.015 \\
\hline \multirow[t]{2}{*}{ GIIIC1 } & 0.001 & 0.279 & $\mathrm{DE}$ & 2.544 & 1.862 & 1.971 & 3.478 & 2.514 & 0.221 \\
\hline & & & & 2.903 & 1.857 & 1.918 & 3.426 & -2.486 & 0.028 \\
\hline GIIIC2 & 0.081 & 0.309 & FM & 2.608 & 1.911 & 2.608 & 3.500 & 2.403 & 0.154 \\
\hline GIIIC3 & 0.015 & 0.429 & FM & 5.335 & 1.867 & 1.983 & 3.500 & 2.410 & 0.000 \\
\hline GIIIC4 & -0.058 & 0.317 & FiM & 3.235 & 1.921 & 1.974 & 3.449 & -2.516 & 0.014 \\
\hline GIVC1 & 0.119 & 0.122 & $\mathrm{FM}$ & 2.478 & 1.952 & 1.852 & 3.653 & 2.354 & 0.252 \\
\hline GIVC2 & 0.250 & 0.211 & FM & 2.460 & 2.022 & 1.905 & 3.526 & 2.470 & 0.171 \\
\hline \multirow[t]{2}{*}{ GIVC3 } & 0.016 & 0.521 & FM & 5.338 & 1.981 & 1.858 & 3.599 & 2.368 & 0.114 \\
\hline & & & & & & & & & 0.022 \\
\hline GIVC4 & -0.047 & 0.250 & FiM & 3.331 & 1.942 & 1.972 & 3.727 & -2.361 & 0.005 \\
\hline
\end{tabular}

duced moment on the neighboring $\mathrm{O}$ atom. In the ground state, i.e., the FiM configuration of GIIC1 the magnetic moment per supercell is $0.89 \mu_{\mathrm{B}}$ which is in agreement with experimental and previous theoretical studies. ${ }^{11,21}$ Magnetic moments on $\mathrm{Fe}, \mathrm{Co}$, and $\mathrm{O}$ atoms (forming Fe-O-Co magnetic path) are $3.220 \mu_{\mathrm{B}},-2.269 \mu_{\mathrm{B}}$, and $0.074 \mu_{\mathrm{B}}$, respectively. It is interesting to note that in case of FM ground-state configuration (such as GIIIC2, GIVC1, and GIVC2) the neighboring $\mathrm{O}$ atom linking $\mathrm{Fe}$ and $\mathrm{Co}$ is polarized ferromagnetically (positive magnetic moment) with respect to both $\mathrm{Co}$ and $\mathrm{Fe}$ while in case of FiM ground-state configurations (such as GIC1, GIC2, and GIIC2) the induced polarizations in $\mathrm{O}$ is antiferromagnetic (AFM) (negative magnetic moment) with respect to $\mathrm{Co}$ and ferromagnetic with respect to Fe.

Incorporating coulomb " $U$ " at transitional metal $d$ orbital can improve the electronic structure description of the system and to further confirm that the calculated magnetic cou- pling is not a consequence of the approximation to exchange and correlation potential, we have also performed GGA $+U$ calculation for different $U$ values in the range from 2 to $6 \mathrm{eV}$ for the GGA ground-state configuration GIIC1. We found that the coupling strength gets reduced with increased $U$ value but for GIIC1 configuration our qualitative conclusion remains unchanged, i.e., FiM coupling is lower in energy (as given in Table II) than FM. The values of local magnetic moments at $\mathrm{Fe}$ and $\mathrm{Co}$ sites increase with the Coulomb $U$ : from $3.426(U=2)$ to $3.691(U=6)$ for $\mathrm{Fe}$ and from -2.441 $(U=2)$ to $-2.710(U=6)$ for Co. Next we computed the energy difference between FM and FiM states for all the configurations (in groups GI, GII, GIII, and GIV) with incorporating $U$ parameter to GGA, i.e., at $\mathrm{GGA}+U$ level $(U$ $=3.5 \mathrm{eV}$ for Fe $d$ and $U=2.8 \mathrm{eV}$ for $\operatorname{Co} d$ ) to find out if the magnetic coupling remains same. In Fig. 4, we have plotted the $\Delta E\left(E_{\mathrm{FiM}}-E_{\mathrm{FM}}\right)$ for all configurations that we have considered here. $\mathrm{GGA}+U$ calculation alters the result signifi-

TABLE II. The energy difference $\Delta E$ between FiM and FM states $\left(\Delta E=E_{\mathrm{FiM}}-E_{\mathrm{FM}}\right.$ in eV), the average moment at $\mathrm{Fe}$, $\mathrm{Co}$, and $\mathrm{O}$ forming $\mathrm{Fe}-\mathrm{O}-\mathrm{Co}$ magnetic path (in $\mu_{\mathrm{B}}$ ) calculated using $\mathrm{GGA}+U$ method for $\mathrm{Zn}_{46} \mathrm{Fe}_{1} \mathrm{Co}_{1} \mathrm{O}_{48}$ supercell in GIIC1 configuration and compared with GGA result.

\begin{tabular}{lrrrrrrr}
\hline \hline $\mathrm{Zn}_{46} \mathrm{Fe}_{1} \mathrm{Co}_{1} \mathrm{O}_{48}$ (GIIC1) & GGA & $U_{\mathrm{Fe}, \mathrm{Co}}=2$ & $U_{\mathrm{Fe}, \mathrm{Co}}=3$ & $U_{\mathrm{Fe}, \mathrm{Co}}=4$ & $U_{\mathrm{Fe}, \mathrm{Co}}=5$ & $U_{\mathrm{Fe}, \mathrm{Co}}=6$ & $U_{\mathrm{Fe}}=3.5, U_{\mathrm{Co}}=2.8$ \\
\hline$\Delta E$ & -0.175 & -0.067 & -0.058 & -0.052 & -0.043 & -0.028 & -0.054 \\
$\mu_{\mathrm{Fe}}$ & 3.220 & 3.426 & 3.511 & 3.578 & 3.635 & 3.691 & 3.542 \\
$\mu_{\mathrm{Co}}$ & -2.269 & -2.441 & -2.537 & -2.605 & -2.661 & -2.710 & -2.524 \\
$\mu_{\mathrm{O}}$ & -0.066 & -0.019 & 0.003 & 0.011 & 0.021 & 0.016 & -0.012 \\
\hline \hline
\end{tabular}




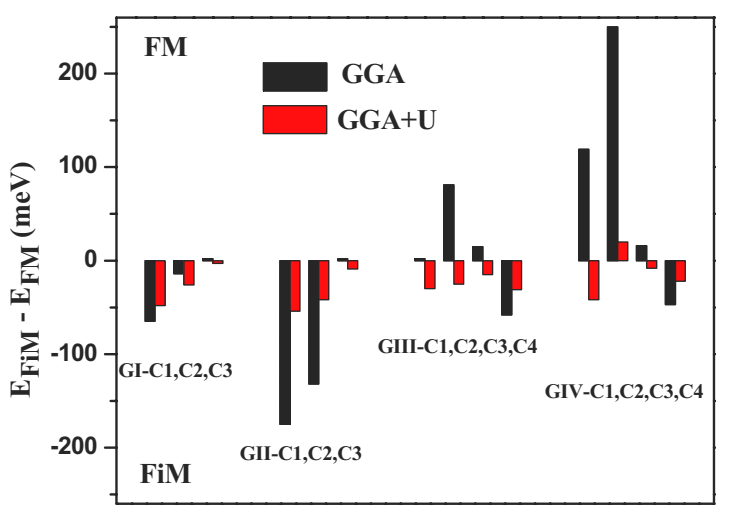

FIG. 4. (Color online) Magnetic exchange interaction energy $\left(\Delta E=E_{\mathrm{FiM}}-E_{\mathrm{FM}}\right.$ in $\left.\mathrm{meV}\right)$ for all the $\mathrm{Fe} / \mathrm{Co}$ codoped configurations based on GGA calculation as shown with black filled bar and $\mathrm{GGA}+U$ calculation as shown as the red (dark gray) filled bar.

cantly. GGA calculation shows in case of surface-subsurface interface (GIII and GIV) first-nearest-neighbor interaction can lead to FM ground state. However in $\mathrm{GGA}+U$ consideration antiferromagnetic superexchange interaction dominates and all configuration leads to FiM ground state except GIVC2 which is weakly ferromagnetic.

In Fig. 5(a) total DOS of the ground state, i.e., GIIC1 in FiM configuration at GGA level has been shown. System has finite DOS at Fermi level and the system is half metallic in nature. The orbital resolved partial DOS at the Fe $3 d$, Co $3 d$, and $\mathrm{O} 2 p$ are plotted in Fig. 5(c). Here we have not found any significant overlap in the DOS between $\mathrm{O} 2 p$ and $\mathrm{Co} 3 d / \mathrm{Fe} 3 d$ and $\mathrm{O}$ atom. This suggest that coupling is not mediated by $\mathrm{O} 2 p$ orbital and therefore the observed FM cannot be described by double exchange mechanism. It is well known that at GGA level the band gap is underestimated, and in case of TM dopant in semiconducting host it also underestimates the static correlation in localized orbital, taking the two factors together electronic structure for this
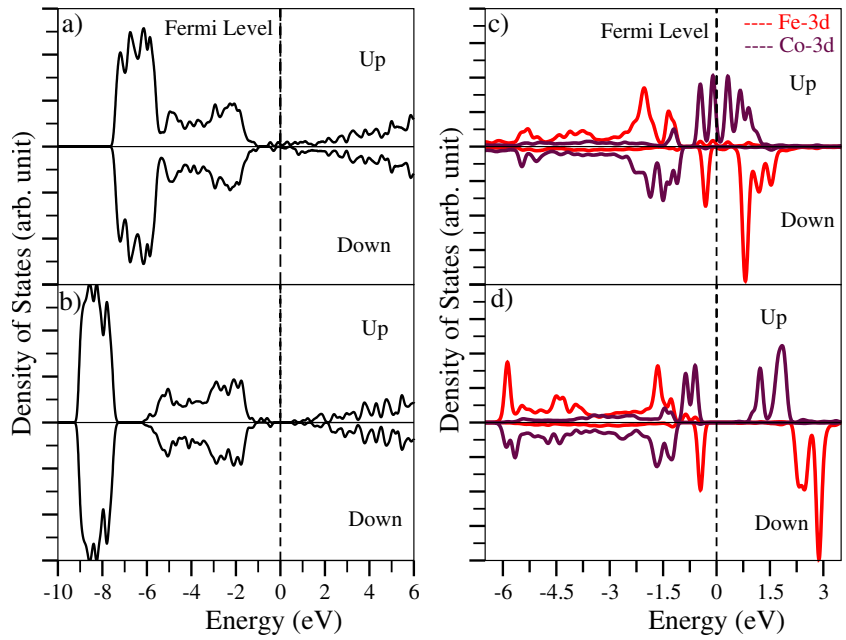

FIG. 5. (Color online) Total and partial density of states for $\mathrm{Fe} / \mathrm{Co}$ doped in GIIC1 configuration calculated using GGA [(a) and (c) $]$ and GGA $+U$ descriptions [(b) and (d)]. Fe $3 d$ and Co $3 d$ partial density of states are shown by red (gray) and maroon (dark) lines, respectively. system leads to the incorrect metallic description. However GGA $+U$ method results in a band gap at Fermi level. The total DOS and partial density of states plot for the ground state, i.e., GIIC1 in FiM configuration for Co at $U=2.8 \mathrm{eV}$ and $\mathrm{Fe}$ at $U=3.5 \mathrm{eV}$ are shown in Figs. 5(b) and 5(d), respectively. Introduction of $U$ separates the partially occupied bands at Fe $d$ and Co $d$ at Fermi level. From DOS [as shown in Fig. 5(d)] for configuration GIIC1 in FiM magnetic state for GGA $+U$ scheme, is clear that $\mathrm{Fe}$ is in +2 state at substitutional $\mathrm{Zn}$ site and favors high spin ground state with the various level serially filled up as $e \uparrow(2), t_{2} \uparrow(3), e \downarrow(1)$, and $t_{2} \downarrow(0)$ (where number in parentheses indicates number of electrons). Also Co is in +2 state when substituted for $\mathrm{Zn}$ site and coupled antiparallel to nearest $\mathrm{Fe}$, favors high spin state with the various level serially filled up as $e \downarrow$ (2), $t_{2} \downarrow$ (3), $e \uparrow(2)$, and $t_{2} \uparrow(0)$. When Coulomb correlation is included in Fe $d$ orbital half-filled $e \downarrow$ band in minority-spin channel (spin down) splits and occupied part pushed down toward valence band (VB) and unoccupied part pushed up inside the conduction band (CB). In case of Co $d$ orbital occupied and unoccupied Co $3 d$ states in majority-spin channel (spin up), i.e., $e \uparrow(2)$ and $t_{2} \uparrow(0)$ states are separated by $\sim 2.1 \mathrm{eV}$. Hence at GGA level the electronic structure leads to metallic description while in case of GGA $+U$ level the occupied and unoccupied parts of $\mathrm{Fe}$ and $\mathrm{Co}$ are separated with proportion of the applied $U$ value. Hence when one $\mathrm{Fe}$ and one Co atom are present in the supercell GGA and $\mathrm{GGA}+U$ calculation reveals that $\mathrm{Fe}$ and Co both preferred to sit on the surface along [0001] direction and coupled antiferromagnetically. While as far as electronic structure is concern $\mathrm{GGA}+U$ description is more realistic than GGA description which is consistence with the previous calculation on $\mathrm{TM}$ doped $\mathrm{ZnO}$ system. ${ }^{21}$

As the ground stat is AFM, the question comes that can we get FM ground state? The possibilities are the following (a) to increase the concentration of $\mathrm{Fe}$ and $\mathrm{Co}$ and to see which magnetic ground state is preferred, i.e., FM, AFM, or FiM, (b) the role of different charge states, and (c) the role of defects, i.e., $\mathrm{Zn}$ vacancy and $\mathrm{O}$ vacancy need to be investigated.

Next we have investigated the effect of concentration of $\mathrm{Fe}$ and $\mathrm{Co}$ impurities in $\mathrm{ZnO}$ nanowire. We replaced two pairs of $\mathrm{Zn}$ atom with $\mathrm{Fe}$ and $\mathrm{Co}$ atoms at different sites taking the GIIC1 (where $\mathrm{Fe}$ and $\mathrm{Co}$ are on cylindrical surface and along [0001] direction) configuration as base and have carried out extensive search for the most favored geometric and magnetic configuration. For this we have considered a longer $\mathrm{ZnO}$ naowire generated from $7 \times 7 \times 4 \mathrm{ZnO}$ supercell, containing a total 192 atoms $\left(\mathrm{Zn}_{96} \mathrm{O}_{96}\right)$. Next we have constructed different configurations where two $\mathrm{Fe}$ and two $\mathrm{Co}$ atoms form nearest neighbors these configurations are defined as near tetramer configurations. To obtain the groundstate geometry and favorable spin alignment in near configurations we have taken converged GIIC1 configuration as the initial geometry. First we have replaced one $\mathrm{Zn}$ by $\mathrm{Fe}$ near to the $\mathrm{Fe}$-Co dimer thus $\mathrm{Fe}$-Co-Fe trimer configurations are generated. We have considered several such possible configurations and calculated the binding energy for each possible configuration. Next separately we have replaced one $\mathrm{Zn}$ by $\mathrm{Co}$ near to $\mathrm{Fe}-\mathrm{Co}$ dimer and hence $\mathrm{Fe}-\mathrm{Co}-\mathrm{Co}$ trimer con- 

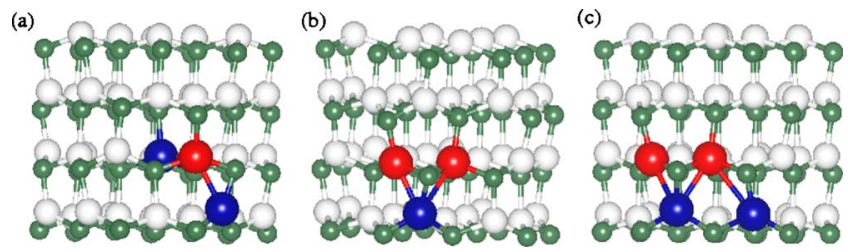

FIG. 6. (Color online) Optimize geometry of (a) FeCoCo, (b) $\mathrm{FeCoFe}$, and (c) $\mathrm{FeCoFeCo}$ clusters in $\mathrm{ZnO}$ nanowire. The white (lighter) spheres are $\mathrm{Zn}$, dark green (gray) small spheres are $\mathrm{O}$, the red (gray) large spheres are $\mathrm{Fe}$, and the blue (dark gray) large spheres are Co.

figurations are generated (as shown in Fig. 6). The formation energy of the defects was calculated using the following expression. $^{22-25}$

We have calculated the formation energy $\left(E^{\mathrm{f}}\right)$ of the different dimer, trimer, and tetramer configurations using the formula,

$$
\begin{aligned}
E^{\mathrm{f}}= & E\left[\mathrm{Zn}_{\mathrm{m}} \mathrm{O}_{\mathrm{n}}(\alpha, q)\right]-E\left[\mathrm{Zn}_{\mathrm{p}} \mathrm{O}_{\mathrm{p}}(\text { pure nanowire })\right] \\
& +\sum n_{\alpha} \mu_{\alpha}+q\left(E_{\mathrm{V}}+\varepsilon_{\mathrm{F}}\right)
\end{aligned}
$$

where $\alpha$ is the defect atom added or removed from the pure nanowire, $n_{\alpha}$ is the number of each defect atoms: $n_{\alpha}$ $=-1(+1)$ for adding (removing) one atom, $E\left[\mathrm{Zn}_{\mathrm{m}} \mathrm{O}_{\mathrm{n}}(\alpha, q)\right]$ is the total energy of the defect in charge state $q$, $E\left[\mathrm{Zn}_{\mathrm{p}} \mathrm{O}_{\mathrm{p}}\right.$ (pure nanowire) $]$ is the total energy of pure $\mathrm{ZnO}$ nanowire, $\mu_{\alpha}$ is the chemical potential of the atom $\alpha$, and $\varepsilon_{\mathrm{F}}$ is Fermi level measured with respect to the valence-band maxima $\left(E_{\mathrm{V}}\right)$ of pure $\mathrm{ZnO}$ nanowire. The chemical potential $\mu$ depends on the experimental growth condition. In thermodynamic equilibrium for bulk system, the $\mathrm{Zn}$ and $\mathrm{O}$ chemical potentials must satisfy the stability condition for $\mathrm{ZnO}$, i.e., $\Delta H(\mathrm{ZnO})_{\text {bulk }}=\mu_{\mathrm{ZnO}}-\left(\mu_{\mathrm{Zn}}^{0}+\mu_{\mathrm{O}}^{0}\right)$, where $\Delta H(\mathrm{ZnO})_{\text {bulk }}$ is the heat of formation in bulk and determined from the computed total energy of bulk wurtize $\mathrm{ZnO}$, hexagonal closed pack $\mathrm{Zn}$, and molecular $\mathrm{O}_{2}$. The calculated heat of formation in bulk is $-3.44 \mathrm{eV}$, which is comparable with the experimental value of $-3.6 \mathrm{eV} .{ }^{26}$ In case of $\mathrm{ZnO}$ nanowire chemical potential has been obtained by a condition that $p\left(\mu_{\mathrm{Zn}}^{0}+\mu_{\mathrm{Zn}}^{\mathrm{x}}+\mu_{\mathrm{O}}^{0}\right.$ $\left.+\mu_{\mathrm{O}}^{\mathrm{x}}\right)=E\left[\mathrm{Zn}_{\mathrm{p}} \mathrm{O}_{\mathrm{p}}(\right.$ pure nanowire $\left.)\right]$, where $\mu_{\mathrm{Zn}}^{\mathrm{x}}$ and $\mu_{\mathrm{O}}^{\mathrm{x}}$ are defined as excess chemical potential for zinc and oxygen, respectively, and governed by the condition that $\mu_{\mathrm{Zn}}^{\mathrm{x}} \leq 0$ and $\mu_{\mathrm{O}}^{\mathrm{x}} \leq 0$ to assure that elementary structure of $\mathrm{Zn}$ and $\mathrm{O}$ have not formed. The extreme $\mathrm{Zn}$-rich (O-poor) condition is given by $\mu_{\mathrm{Zn}}^{\mathrm{x}}=0$ and extreme $\mathrm{O}$-rich (Zn-poor) condition is given by $\mu_{\mathrm{O}}^{\mathrm{x}}=0$. For Fe and Co we take both $\mu_{\mathrm{Fe}}^{\mathrm{x}}=0$ and $\mu_{\mathrm{Co}}^{\mathrm{x}}=0$. Chemical potential for $\mathrm{Fe}$ is calculated as the energy per $\mathrm{Fe}$ atom of cubic crystal structure and chemical potential for Co is calculated as energy per Co of hcp crystal structure. All formation energies are calculated using GGA $+U$ method.

From Fig. 7(a), we find that formation energy of substitutional pair Fe-Co in neutral charge state (GIIC1 configuration in FiM ground state) in $\mathrm{Zn}$-rich condition is $3.57 \mathrm{eV}$ while in $\mathrm{O}$ rich the value is $-0.98 \mathrm{eV}$. According to the definition positive value of formation energy means energy is required to incorporate $\mathrm{Fe} / \mathrm{Co}$ in $\mathrm{ZnO}$ nanowire. Thus the formation of $\mathrm{Fe}-\mathrm{Co}$ defect is more likely to be formed in O-rich condition.

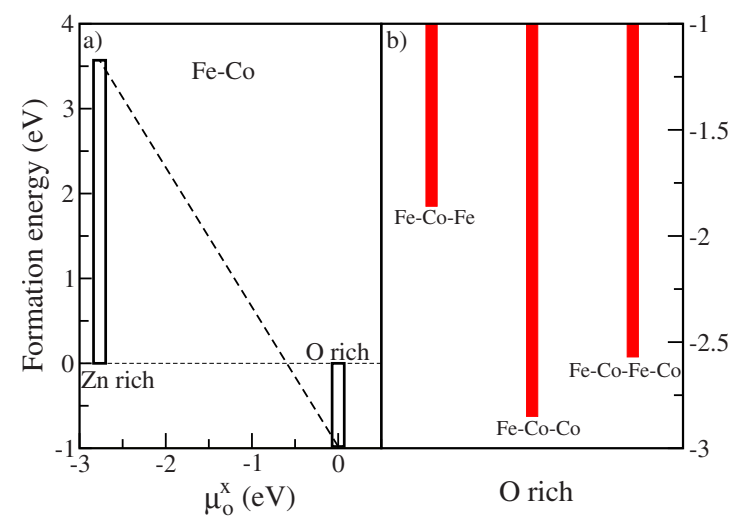

FIG. 7. (Color online) (a) Variation in formation energy of $\mathrm{Fe}-\mathrm{Co}$ defect in $\mathrm{ZnO}$ nanowire with oxygen excess chemical potential at both $\mathrm{Zn}$-rich and O-rich growth conditions, as shown by unfilled bars and (b) comparison of formation energy of $\mathrm{Fe}-\mathrm{Co}$, $\mathrm{Fe}-\mathrm{Co}-\mathrm{Co}$, and $\mathrm{Fe}-\mathrm{Co}-\mathrm{Fe}-\mathrm{Co}$ defect configurations at O-rich growth condition, shown as filled red (gray) bar.

In Fig. 7(b), we have shown the formation of all other trimer and tetramer substitutional defects in O-rich condition with respect to $\mathrm{Fe}-\mathrm{Co}$ formation energy in O-rich condition. The ground-state $\mathrm{Fe}-\mathrm{Co}$, Fe-Co-Fe trimer, Fe-Co-Co trimer, and Fe-Co-Fe-Co tetramer configurations are shown Fig. 5. Formation energy $\mathrm{Fe}-\mathrm{Co}-\mathrm{Fe}$ trimer, $\mathrm{Fe}-\mathrm{Co}-\mathrm{Co}$ trimer, and $\mathrm{Fe}-$ Co-Fe-Co tetramer (near) configurations are 1.86, 2.85, and $2.57 \mathrm{eV}$ lower in energy that Fe-Co pair formation energy. This shows that the $E^{\mathrm{f}}$ of trimer configurations are less compare with dimer, hence less energy will be required to substitute Fe/Co atoms for $\mathrm{Zn}$ close to Fe-Co dimer. Thus third impurity wants to form trimer cluster with the Fe-Co dimer on the cylindrical surface in $\mathrm{ZnO}$ nanowire. The ground-state spin configuration for both $\mathrm{Fe}-\mathrm{Co}-\mathrm{Fe}$ and $\mathrm{Fe}-\mathrm{Co}-\mathrm{Co}$ is ferrimagnetic $\left(\uparrow \downarrow \uparrow ;\right.$ FiM) with net magnetic moment of $5.0 \mu_{\mathrm{B}}$ and $4.0 \mu_{\mathrm{B}}$, respectively. This shows that $\mathrm{Fe}$ atoms are tend to couple ferromagnetically whereas Co atoms tend to couple antiferromagetically.

To investigate the formation of tetramer cluster keeping $\mathrm{Fe}$ and $\mathrm{Co}$ concentration same, first we have taken the all $\mathrm{Fe}-\mathrm{Co}-\mathrm{Fe}$ configurations and replace one $\mathrm{Zn}$ near to it by $\mathrm{Co}$ it this leads to Fe-Co-Fe-Co tetramer configurations. Similarly taking $\mathrm{Fe}-\mathrm{Co}-\mathrm{Co}$ configurations and substituting one $\mathrm{Zn}$ near to the defect by Fe leads to Fe-Co-Co-Fe tetramer configurations. It is interesting to note that the ground-state geometry is a Fe-Co-Fe-Co tetramer configuration on the cylindrical surface of $\mathrm{ZnO}$ naowire and consists of two Fe-Co dimers and have lowest formation energy when compared with other tetramer cluster configurations. Further we have consider the configuration where two pairs of Fe-Co dimers are on the surface along [0001] direction and are $10.45 \AA$ apart from each other. This configuration is generated by doubling the GIIC1 unit cell and defined as far configuration. This configuration is $0.85 \mathrm{eV}$ higher in energy than near tetramer configuration which implies that $\mathrm{Fe}-\mathrm{Co}$ has a tendency to form cluster. Also it is interesting to note that the formation energy of $\mathrm{Fe}-\mathrm{Co}-\mathrm{Co}$ trimer configuration is lower in energy when compare to $\mathrm{Fe}-\mathrm{Co}-\mathrm{Fe}$ trimer and $\mathrm{Fe}-\mathrm{Co}-$ $\mathrm{Fe}-\mathrm{Co}$ tetramer configurations. This implies that it is difficult 

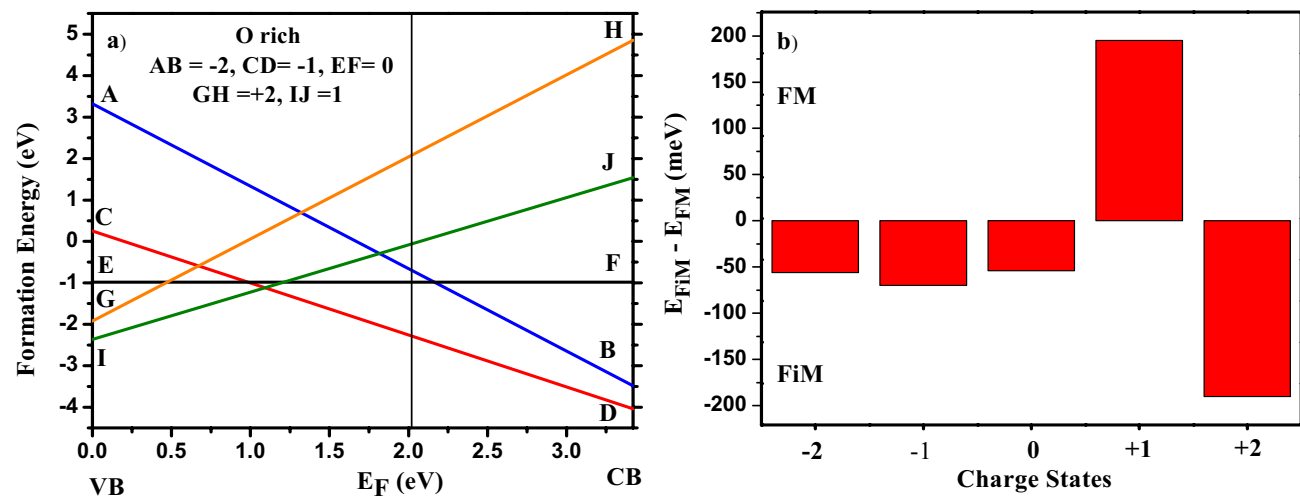

FIG. 8. (Color online) (a) Formation energy of different charge state of Fe-Co defect in ZnO nanowire at O-rich growth condition. Blue $(\mathrm{AB})$, red $(\mathrm{CD})$, black $(\mathrm{EF})$, orange $(\mathrm{GH})$, and green $(\mathrm{IJ})$ line represent variation in formation energy for charge states $-2,-1,0,+2$, and +1 , respectively, and (b) magnetic exchange interaction energy $\left[\Delta E=E_{\mathrm{FiM}}-E_{\mathrm{FM}}\right]$ for different charge state of $\mathrm{Fe}-\mathrm{Co}$ defect in $\mathrm{ZnO}$ nanowire. Shown as red (gray) filled bar.

to keep the same type of atoms away from each other and there is a strong tendency that Co atoms will try to form cluster around a $\mathrm{Fe}$ atom which will suppress the ferromagnetic coupling. Previous density functional calculations also shows that Co have a strong clustering tendency when incorporated in $\mathrm{ZnO}$ matrix. ${ }^{27}$ This tendency will form separate magnetic centers of Fe, Co clusters of different Fe,Co concentration. It will be interesting to investigate how these magnetic centers interact with each other; on the other hand experimentally it is still not clear that how frequent the different transition metals form nearest neighbors sites when compare with the same type in codoped case.

The favorable spin state of Fe-Co-Fe-Co tetramer configuration is ferrimagnetic coupling $(\uparrow \downarrow \uparrow \downarrow$; FiM), which has a total magnetic moment of $2.00 \mu_{\mathrm{B}}$. The magnetic moment on each atom is $3.556,-2.482,3.507$, and -2.525 . The $(\uparrow \downarrow \uparrow \downarrow ;$ FiM) spin state is $0.096 \mathrm{eV}, 0.031 \mathrm{eV}, 0.281 \mathrm{eV}$, $0.358 \mathrm{eV}, 0.338 \mathrm{eV}$, and $0.059 \mathrm{eV}$ lower in energy than $(\uparrow \uparrow \uparrow \uparrow ; \mathrm{FM}),(\uparrow \uparrow \downarrow \downarrow ; \mathrm{AFM}),(\downarrow \uparrow \uparrow \uparrow ; \mathrm{FiM}),(\uparrow \downarrow \uparrow \uparrow:$ FiM $)$, $(\uparrow \uparrow \downarrow \uparrow ;$ FiM), and $(\uparrow \uparrow \uparrow \downarrow ;$ FiM) states, respectively. From the above discussion we can conclude that FiM is energetically preferred over FM state for Fe/Co clusters for more than two $\mathrm{Fe} / \mathrm{Co}$ atoms. Hence from the preceding discussion shows that $\mathrm{Fe} / \mathrm{Co}$ codoped $\mathrm{ZnO}$ nanowire is unlike to stabilize in ferromagnetic ground state.

As shift of the Fermi level of Fe-Co doped $\mathrm{ZnO}$ nanowire with respect to pure nanowire reveals that impurities lead to an electronic doping effect, hence we have considered different charge state of $\mathrm{Fe} / \mathrm{Co}$ defect in $\mathrm{ZnO}$ nanowire and find out which is the most stable under varying condition. Formation energies of all the charge states of Fe-Co defect at both O-rich growth conditions are shown in Fig. 8(a) and corresponding magnetic exchange energies (i.e., $E_{\mathrm{FM}}$ vs $\left.E_{\mathrm{FiM}}\right)$ in Fig. 8(b). We found that +1 charge state of the defect can promote FM.

From the preceding discussion we can conclude that in $\mathrm{Fe} / \mathrm{Co}$ codoped $\mathrm{ZnO}$ nanowire intrinsic $\mathrm{FM}$ is unlike and additional carriers are needed to promote FM. In order to understand the observed FM in recent experiment by Liu et $a l .{ }^{6}$ on $\mathrm{Fe} / \mathrm{Co}$ codoped $\mathrm{ZnO}$ nanowires we also explored the possibility of carrier mediated exchange interaction via (a)
$\mathrm{Zn}$ vacancy or (b) O vacancy. In case of vacancy calculations neutral as well as charge states of the defect complex have been considered in GIICI defect configuration and geometry optimization for both FM and FiM for all the cases have been performed by $\mathrm{GGA}+U$. In case of $\mathrm{Zn}$ vacancy, vacancy sites has been considered near to the Fe-Co defect while in case of $\mathrm{O}$ vacancy we have considered $\mathrm{O}$ vacancy far from the defect and $\mathrm{Fe}-\mathrm{V}_{\mathrm{O}}-\mathrm{Co}$ defect configuration created by removing $\mathrm{O}$ atom from $\mathrm{Fe}-\mathrm{O}-\mathrm{Co}$ magnetic path. Here by far, we mean at such distance we can have considerable magnetic coupling, if a defect is created too far from the $\mathrm{Fe}-\mathrm{Co}$ pair then by using first-principles tools we will not get considerable coupling and $\Delta E$, i.e., $E_{\mathrm{AFM}}-E_{\mathrm{FM}}$ will near equal to zero; also in case of Group-I, i.e., when Fe-Co pair is in the bulk we have considered the $\mathrm{V}_{\mathrm{O}}$ vacancy defect in the bulk and for GII, GIII, and GIV configurations, i.e., when Fe-Co pair in on the surface or surface-subsurface interface we have created the defect on the surface. For the defect complexes we have considered all the possible charge state and we have tried to find out which is more favorable under varying condition. In Figs. 9(a) and 9(b) we have shown the formation energies of all the defect complexes in for O-rich growth condition and $\mathrm{Zn}$-rich growth condition, respectively. In Fig. 10 , corresponding magnetic exchange energies (i.e., $E_{\mathrm{FM}} \mathrm{vs}$ $\left.E_{\mathrm{FiM}}\right)$ are shown for the configuration GIICI with $\mathrm{Zn}$ vacancy (near), $\mathrm{O}$ vacancy (far and on the surface), and $\mathrm{Fe}-\mathrm{V}_{\mathrm{O}}-\mathrm{Co}$ defect complex, respectively.

In case of $\mathrm{Zn}$ vacancy we have not found any FM ground state in GIICI configuration. Interestingly $\mathrm{O}$ vacancy far from the defect in GIICI configuration is FiM while for $\mathrm{Fe}-\mathrm{V}_{\mathrm{O}}-\mathrm{Co}$ defect complex we have $\mathrm{FM}$ coupling for all charge states. However which one of these defect complexes is favorable in terms of formation energy that depends on the growth condition. In O-rich growth condition Zn-vacancy defects are lower in formation energy and in Zn-rich growth condition $\mathrm{O}$ vacancy related defects are lower in formation energy. The formation energy of neutral $\mathrm{O}$ vacancy at far has lower formation energy compare to $\mathrm{Fe}-\mathrm{V}_{\mathrm{O}}-\mathrm{Co}$ defect complex; however it is interesting to note that for +1 and +2 charge state $\mathrm{Fe}-\mathrm{V}_{\mathrm{O}}-\mathrm{Co}$ defect is lower in formation energy at $\mathrm{VB}$ site and -1 and -2 charge state of $\mathrm{Fe}-\mathrm{V}_{\mathrm{O}}-\mathrm{Co}$ is lower in 

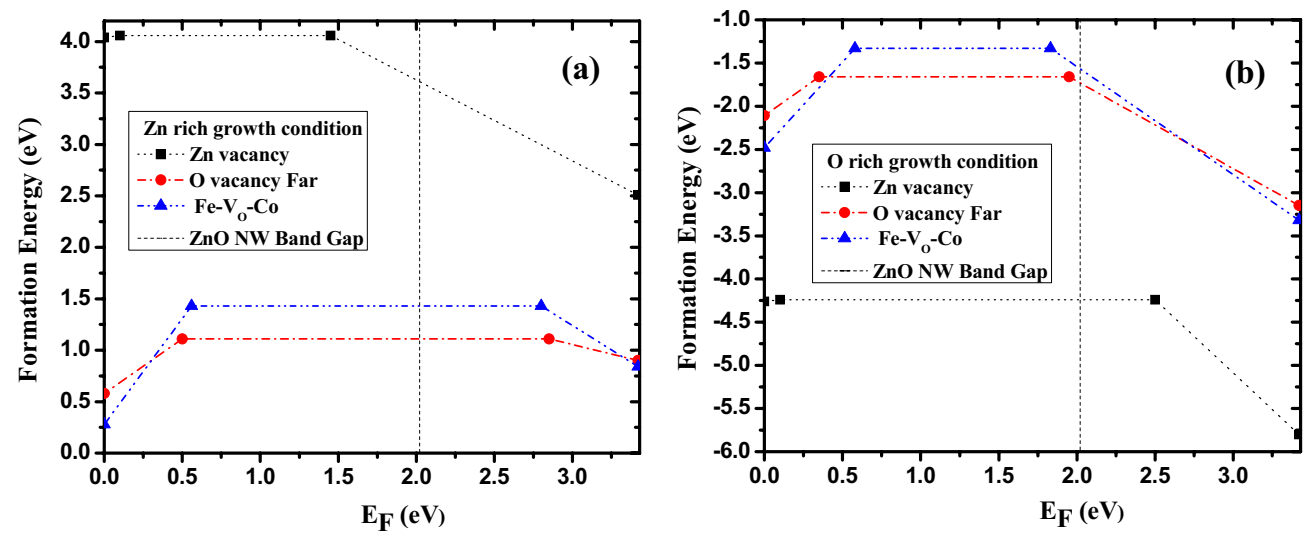

FIG. 9. (Color online) Formation energy of Zn vacancy, O vacancy far, and Fe-V $\mathrm{V}_{\mathrm{O}^{-}}$Co defect at both (a) Zn-rich and (b) O-rich growth conditions. Lowest formation energies are only plotted. $\mathrm{Zn}$ vacancy, $\mathrm{O}$ vacancy far, and $\mathrm{Fe}-\mathrm{V}_{\mathrm{O}}-\mathrm{Co}$ defect complexes are shown by black, red (gray), and blue (dark) lines, respectively.

formation energy at CB site for both Zn-rich and O-rich growth conditions compare with $\mathrm{O}$ vacancy far, however in the later case the difference is small. Thus in $\mathrm{Zn}$-rich growth condition at $p$-type (VB side) doping condition the observed $\mathrm{FM}$ is due to the direct exchange of holes in $\mathrm{Fe}-\mathrm{V}_{\mathrm{O}}$-Co defect complex and in $n$-type ( $\mathrm{CB}$ side) doping condition the FM state can be stable by direct exchange of electrons. On the other hand at O-rich growth condition $\mathrm{Zn}$ vacancy with +1 charge state is the lowest value of formation energy at $p$-type (VB side) doping condition and -1 charge state is the lowest value of formation energy at $n$-type (CB side) doping condition. However we have not found any FM ordering due to Zn-vacancy related defect in GIIC1 configuration.

The formation energies of different defect complex with varying charge state for different growth conditions are expected to vary with different location of Fe-Co pair. Which one of the defect complex is favorable under varying condition will depend on the fact that where Fe-Co pair is formed,

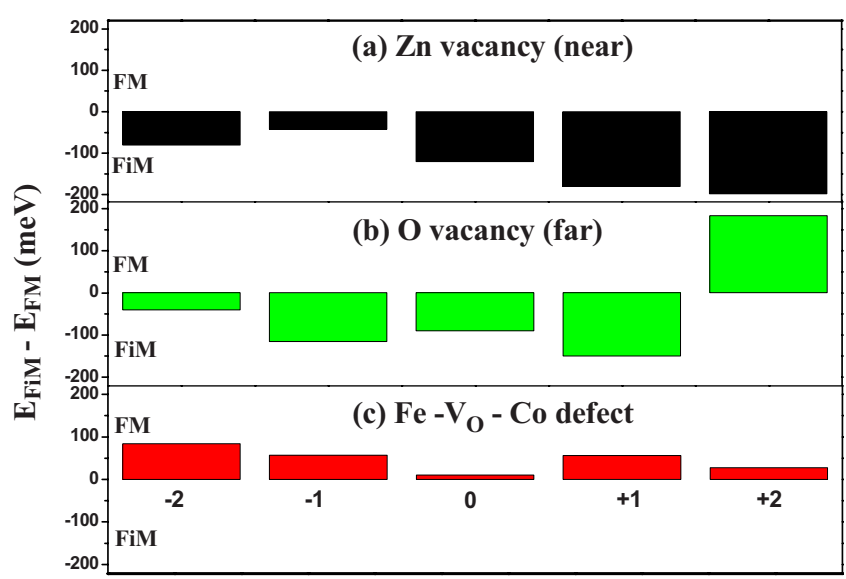

Charge States

FIG. 10. (Color online) Magnetic exchange interaction energy $\left[\Delta E=E_{\mathrm{FiM}}-E_{\mathrm{FM}}\right]$ of different charge state of $\mathrm{Zn}$ vacany, $\mathrm{O}$ vacany far, and $\mathrm{Fe}-\mathrm{V}_{\mathrm{O}^{-}}$-Co defect for GIIC1 configuration. Black (black), green (light gray), and red (dark gray) filled bars represents $\mathrm{Zn}$ vacancy, $\mathrm{O}$ vacancy far, and $\mathrm{Fe}-\mathrm{V}_{\mathrm{O}}-\mathrm{Co}$ defect complex, respectively. i.e., on the surface, surface-subsurface interface, or in bulk environment. In this communication we have considered the formation of vacancy related defects only for the situation where $\mathrm{Fe}-\mathrm{Co}$ pair is on the surface and concluded that on the surface $\mathrm{Fe}-\mathrm{V}_{\mathrm{O}}-\mathrm{Co}$ charge state defects complexes have lower formation energy compare to $\mathrm{O}$ vacancy created at far.

Next we have extended our study for $\mathrm{O}$ vacancy far and $\mathrm{Fe}-\mathrm{V}_{\mathrm{O}}-\mathrm{Co}$ in neutral charge state for all configurations of Fe-Co pair. In Figs. 11(a) and 11(b) variation magnetic exchange energy $(\Delta E)$ with Fe-Co all the configurations are shown for both $\mathrm{O}$-vacancies at far and $\mathrm{Fe}-\mathrm{V}_{\mathrm{O}}-\mathrm{Co}$ defect complex at neutral charge state. In case of $\mathrm{O}$ vacancy far we have an oscillatory variation, i.e., the sign of the coupling is altered with the Fe-Co separation, i.e., this sign of RKKY type of exchange interaction for the group GI, i.e., when $\mathrm{Fe}-\mathrm{Co}$ pair is in bulk environment and the $\mathrm{O}$ vacancy in created in the bulk. However in case of Fe-Co pair on the surface of the preferred magnetic coupling is always remain FiM when $\mathrm{O}$ vacancy is far from the defect and on the surface. We found that $\mathrm{Fe}-\mathrm{V}_{\mathrm{O}}-\mathrm{Co}$ defect configurations (formed by removing the $\mathrm{O}$ atom from the $\mathrm{Fe}-\mathrm{O}-\mathrm{Co}$ magnetic path) are ferromagnetic when Co-Fe separation is less than $2.596 \AA$ in GGA scheme and $2.801 \AA$ in GGA $+U$ scheme irrespective of the location of transition-metal ions. In case of Fe- $\mathrm{V}_{\mathrm{O}}-\mathrm{Co}$ defect configurations the $\mathrm{Fe}-\mathrm{Co}$ bond distances are reduced compared to the Fe-O-Co defect configurations. Previous firstprinciples calculation reported that Fe-Co pairs are ferromagnetic with a separation of $2.1 \AA^{28,29}$ Hence these results indicate that the competition of RKKY exchange interaction and direct exchange via $\mathrm{Fe}-\mathrm{V}_{\mathrm{O}}-\mathrm{Co}$ is the main competing factor for stabilization of $\mathrm{FM}$ in $\mathrm{Fe}-\mathrm{Co}$ codoped $\mathrm{ZnO}$ nanowire.

\section{SUMMARY}

In conclusion, we have shown that in $\mathrm{Fe} / \mathrm{Co}$ codoped $\mathrm{ZnO}$ nanowire substitution of $\mathrm{Zn}$ by $\mathrm{Fe}$ and $\mathrm{Co}$ is favorable in $\mathrm{O}$-rich growth condition. The ground-state geometry is found as where $\mathrm{Fe}$ and $\mathrm{Co}$ are nearest neighbors on the surface along [0001] direction and corresponding magnetic ground state is FiM. Considering all configuration we found that, the 
(a) O vacancy (Far)

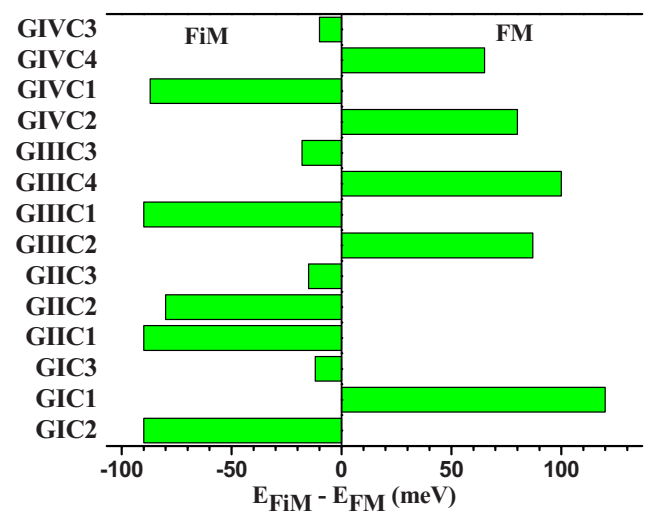

(b) $\mathrm{Fe}-\mathrm{V}_{\mathrm{O}}$ - Co defect

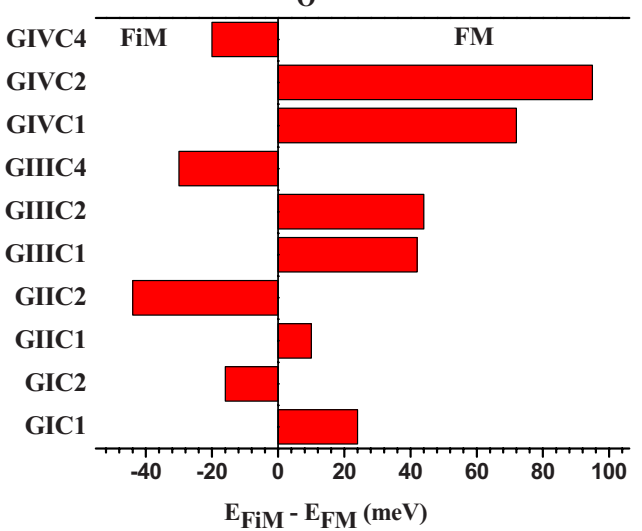

FIG. 11. (Color online) Magnetic exchange interaction energy $\left[\Delta E=E_{\mathrm{FiM}}-E_{\mathrm{FM}}\right]$ for (a) $\mathrm{O}$ vacancy far, shown as green (light gray) filled bars and (b) $\mathrm{Fe}-\mathrm{V}_{\mathrm{O}}$-Co defect, shown as red (dark gray) filled bars for all Fe/Co coped configurations as shown in Fig. 2.

spin alignment between two impurity atoms depends on their location and radial confinement. In GGA description ferromagnetic ordering is observed when impurity atoms sit at surface and subsurface interface forming Fe-O-Co magnetic path. However the magnet ground state altered significantly when $\mathrm{GGA}+U$ description is considered. In case of $\mathrm{Fe}-\mathrm{Co}$ doped $\mathrm{ZnO}$ nanowire in neutral charge state the short-range FiM interaction is dominant over double exchange and the magnetic ground state is FiM. Also it is difficult to keep the same type of atoms away from each other and there is a strong tendency that Co atoms will try to from cluster around a $\mathrm{Fe}$ atom which will lead to inhomogeneous concentration of $\mathrm{Fe}$ and $\mathrm{Co}$ and FiM coupling will be the preferred magnetic aliment. When two $\mathrm{Fe}$ and $\mathrm{Co}$ atoms are considered in the supercell keeping Fe,Co concentration same, the Fe-Co dimer forms cluster on the surface of the nanowire with FiM as magnetic ground state. Intrinsically the system in ferrimagnetic can be altered to fermomagnetic by addition of one hole per Fe-Co pair. In case vacancy related defect when considered ground-state $\mathrm{Fe}-\mathrm{Co}$ pair configuration where $\mathrm{Fe}-\mathrm{Co}$ is on the surface, we found only particular defect complex, i.e., Fe- $\mathrm{V}_{\mathrm{O}}-\mathrm{Co}$ is FM. The formation of defect is depends on the growth condition and expected to depend on the location of $\mathrm{Fe}-\mathrm{Co}$ pair as well. In case of $\mathrm{O}$ vacancy related defect we have two distinct mechanisms which can lead to FM (the two competing mechanism), the RKKY type of exchange interaction in bulk environment and direct exchange interaction of carriers due to $\mathrm{Fe}-\mathrm{V}_{\mathrm{O}}-\mathrm{Co}$ defect configuration on the surface.

\section{ACKNOWLEDGMENTS}

We thank the staff of the Center of Computational Materials Science at IMR for the use of Hitachi SR11000-K2 Supercomputing facilities. G.P.D. acknowledges the support from the BRNS (India) under the Spintronics CRP.
*Corresponding author; saurabhghosh2802@gmail.com

${ }^{1}$ S. A. Wolf, D. D. Awschalom, R. A. Buhrman, J. M. Daughton, S. Von Molnar, M. L. Roukes, A. Y. Chtchelkanova, and D. M. Treger, Science 294, 1488 (2001).

${ }^{2}$ D. M. Bagnall, Y. F. Chen, Z. Zhu, T. Yao, S. Koyama, M. Y. Shen, and T. Goto, Appl. Phys. Lett. 70, 2230 (1997).

${ }^{3}$ Z. K. Tang, G. K. L. Wong, P. Yu, M. Kawasaki, A. Ohtomo, H. Koinuma, and Y. Segawa, Appl. Phys. Lett. 72, 3270 (1998).

${ }^{4}$ W. B. Jian, Z. Y. Wu, R. T. Huang, F. R. Chen, J. J. Kai, C. Y. Wu, S. J. Chiang, M. D. Lan, and J. J. Lin, Phys. Rev. B 73, 233308 (2006).

${ }^{5}$ J. J. Wu, S. C. Liu, and M. H. Yang, Appl. Phys. Lett. 85, 1027 (2004).

${ }^{6}$ L. Q. Liu, B. Xiang, X. Z. Zhang, Y. Zhang, and D. P. Yu, Appl. Phys. Lett. 88, 063104 (2006).

${ }^{7}$ J. J. Chen, M. H. Yu, W. L. Zhou, K. Sun, and L. M. Wang, Appl. Phys. Lett. 87, 173119 (2005).

${ }^{8}$ J. J. Liu, K. Wang, M. H. Yu, and W. L. Zhou, J. Appl. Phys. 102, 024301 (2007).
${ }^{9}$ X. L. Zhang, R. Qiao, R. Qiu, Y. Li, and Y. S. Kang, J. Phys. Chem. A 111, 4195 (2007).

${ }^{10}$ Y. M. Cho, W. K. Choo, H. Kim, D. Kim, and Y. E. Ihm, Appl. Phys. Lett. 80, 3358 (2002).

${ }^{11}$ M. S. Park and B. I. Min, Phys. Rev. B 68, 224436 (2003).

${ }^{12}$ Q. Wang, Q. Sun, G. Chen, Y. Kawazoe, and P. Jena, Phys. Rev. B 77, 205411 (2008).

${ }^{13}$ W. Kohn and L. J. Sham, Phys. Rev. 140, A1133 (1965).

${ }^{14}$ Y. Wang and J. P. Perdew, Phys. Rev. B 44, 13298 (1991).

${ }^{15}$ G. Kresse and J. Furthmuller, Phys. Rev. B 54, 11169 (1996).

${ }^{16}$ G. Kresse and D. Joubert, Phys. Rev. B 59, 1758 (1999).

${ }^{17}$ Q. Wang and P. Jena, Appl. Phys. Lett. 84, 4170 (2004).

${ }^{18}$ Q. Wang, Q. Sun, Y. Kawazoe, and P. Jena, Appl. Phys. Lett. 87, 162509 (2005).

${ }^{19}$ S. Lany, H. Raebiger, and A. Zunger, Phys. Rev. B 77, 241201(R) (2008).

${ }^{20}$ A. Walsh, J. L. F. Da Silva, and S.-H. Wei, Phys. Rev. Lett. 100, 256401 (2008).

${ }^{21}$ P. Gopal and N. A. Spaldin, Phys. Rev. B 74, 094418 (2006). 
${ }^{22}$ S. Ossicini, E. Degoli, F. Iori, E. Luppi, R. Magri, G. Cantele, F. Trani, and D. Ninno, Appl. Phys. Lett. 87, 173120 (2005).

${ }^{23}$ P. Mahadevan and A. Zunger, Phys. Rev. Lett. 88, 047205 (2002).

${ }^{24}$ C. G. Van de Walle and J. Neugebauer, J. Appl. Phys. 95, 3851 (2004).

${ }^{25}$ N. Ganguli, I. Dasgupta, and B. Sanyal, Appl. Phys. Lett. 94, 192503 (2009).
${ }^{26}$ F. Tuomisto, K. Saarinen, D. C. Look, and G. C. Farlow, Phys. Rev. B 72, 085206 (2005).

${ }^{27}$ D. Iuşan, M. Kabir, O. Grånäs, O. Eriksson, and B. Sanyal, Phys. Rev. B 79, 125202 (2009).

${ }^{28}$ A. N. Andriotis, G. Mpourmpakis, G. E. Froudakis, and M. Menon, J. Chem. Phys. 120, 11901 (2004).

${ }^{29}$ G. Mpourmpakis, G. E. Froudakis, A. N. Andriotis, and M. Menon, Phys. Rev. B 72, 104417 (2005). 